\title{
Interactions éducatives en contexte plurilingue et multiculturel
}

\author{
Rodica Ailincai \\ MoDyCo (Modèles, Dynamiques, Corpus - Université Paris 10 bâtiment A - Bureau 402 A 200, Avenue De La \\ Republique 92001 Nanterre Cedex) \\ r.ailincai@gmail.com
}

\section{Introduction}

Les derniers travaux sur les interactions à visée d'apprentissage ont mis en évidence des cadres d'analyse qui proposent de prendre en compte l'ensemble des déterminants (d'ordre épistémologique, cognitif, développemental et conversationnel) impliqués dans les apprentissages, afin d'articuler par une approche descriptive et explicative le cognitif du sujet, le savoir en jeu et les pratiques conversationnelles. Nous pensons que les différences et les similitudes individuelles et culturelles relatives aux interactions éducatives restent insuffisamment explorées en France, les cadres et modèles existants ne suffisant pas à rendre compte de la dynamique interactionnelle en contexte pluriculturel et plurilingue.

L'objectif premier de cette recherche à caractère exploratoire, est de proposer un modèle d'analyse différentielle de l'interaction à visée d'apprentissage qui sera à la fois descriptif, explicatif et compréhensif. Pour ce faire, nous nous proposons d'étudier, à côté des facteurs cités, le déterminant d'ordre culturel(1), dans une approche comparative(2), en reconsidérant la place de l'intervention non verbale au sein de l'interaction communicative(3).

Le contexte de la Guyane, vrai laboratoire expérimental sur le plan du plurilinguisme et de l'interculturalisme, nous a semblé un terrain pertinent pour cette recherche empirique dont nous présentons ici les premiers résultats; ainsi, l'analyse des interactions éducatives abordée dans cette contribution concerne deux des six classes d'élèves de grande section que la recherche se propose d'étudier (classes qui sont situées sur des sites isolés, éloignés les uns des autres et présentant des spécificités linguistiques et culturelles).

Le modèle d'analyse que nous nous proposons de réaliser permettra de rendre compte à travers une approche interculturaliste des échanges et négociations dans le cadre des apprentissages scolaires en contexte plurilingue. Par ailleurs en dehors des objectifs scientifiques cités ci-dessus cette recherche répond à des besoins impératifs en termes de réussite scolaire, le système éducatif français se montrant peu performant dans l'académie de la Guyane. Les facteurs explicatifs ont trait aux aspects que nous nous proposons d'aborder dans cette recherche, (a) l'allophonie des élèves ( $28 \%$ d'élèves issus de la migration, mais aussi de nombreux élèves français-alloglottes) et (b) la formation des enseignants (souvent de passage et/ou non qualifiés) :

- Il existe aujourd'hui de nombreuses publications portant sur la situation sociolinguistique guyanaise (Leconte \& Caitucoli, 2003 ; Léglise \& Migge, 2007 ; Léglise, 2004, 2005, 2007), et un certain nombre d'entre elles sur la relation que l'école entretient avec ce plurilinguisme (Alby \& Léglise, 2006, 2007b ; Léglise \& Puren, 2005). Elles mettent toutes en évidence la particularité du contexte guyanais de ce point de vue : "on dénombre plus d'une trentaine de langues en Guyane. Les unes et les autres pesant un poids - numérique, économique, symbolique, etc. plus ou moins important. Sur cette trentaine de langues, [...] une vingtaine est parlée par des groupes de locuteurs - «natifs » ou non - représentant plus de 1\% de la population. » Léglise (2007, p.39).

- Cette diversité sociolinguistique associée à l'insuffisante préparation des enseignants majoritairement métropolitains à intervenir dans le contexte mentionné ainsi qu'aux difficultés 
matérielles d'une partie importante de population (liées à la dispersion sur le territoire) sont des facteurs qui impactent sur la réussite scolaire en Guyane.

Les recherches concernant la réussite scolaire soulignent depuis longtemps l'importance des représentations que le sujet se construit autour de l'école, représentations qui ne sont pas les mêmes d'une culture à l'autre et qui, chez l'enfant «primo arrivant », qui aborde l'apprentissage initial, viennent de la famille, mais aussi de l'école, des pairs, des médias. Le poids qu'exerce la famille sur le développement et l'adaptation scolaire de l'enfant est considérable : les réalités familiales (comportements, attitudes, traits de personnalité et potentiel intellectuel des parents, statut et environnement social) peuvent expliquer plus de $84 \%$ de la variance des acquisitions scolaires (Pourtois, 1979). En référence à un modèle écologique et culturel du développement proposé par Ogbu (1981), il est possible de considérer que les conceptions jouent un rôle sur la manière dont les familles et les enfants vont investir les activités scolaires ${ }^{1}$.

Ces données interculturelles attirent l'attention sur les différences d'ordre représentationnel qui interviennent quant à la manière dont les enfants investissent les apprentissages scolaires.

L'intérêt qui porte notre recherche à l'étude des interactions en contexte plurilingue et multiculturel et au rôle des représentations afférentes aux domaines de connaissances se justifie d'une part par le déficit d'études dans le domaine et d'autre part par la nécessité de comprendre les investissements différentiels des enfants selon leur groupe d'appartenance.

\section{Cadre théorique et problématique}

En se situant dans un cadre théorique référant à l'interactionnisme social, cognitif et au socioconstructivisme nous nous interrogeons sur la nature des interactions à visée d'apprentissage en contexte scolaire sous deux angles complémentaires: (a) d'une part l'analyse se focalisera sur les interactions à visée d'apprentissage des sciences, (b) d'autre part sur les interactions à visée d'apprentissage des langues. Par l'appellation apprentissages scolaires ont entend les apprentissages qui relèvent de processus sociaux très contrôlés du point de vue des contenus d'apprentissages (les programmes), des agents qui les transmettent (les enseignants), des pratiques pédagogiques (méthodes), des modes de validation (l'évaluation), des lieux où ils se déroulent(les écoles (Weil-Barais, 2004).

Nous rendrons compte de la structure de l'interaction à visé d'apprentissage en faisant référence à ses diverses formes : un accompagnement de tutelle dans la réalisation d'une tâche (ou « étayage » selon Bruner 1972), un accompagnement type médiation sémiotique (pour déployer la zone de proche développement de l'apprenant, Vygotski 1934), un accompagnement dans les sauts épistémologiques nécessaires à la démarche scientifique (ou « obstacles » chez Bachelard 1938).

L'adoption d'un point de vue Vygotskien conduit à considérer que les dispositifs éducatifs incitant les enfants à construire des représentations de la réalité d'un niveau supérieur à celui dont ils disposent déjà (interaction asymétrique) contribueraient à stimuler leur développement au plan intellectuel.

Les travaux de Bruner nous intéressent également pour l'approche entre la psychologie cognitive et la psychologie culturelle : pour lui, l'adulte agit comme un médiateur de la culture; la culture va apporter à l'enfant toutes les bases de son développement cognitif. Pour lui le développement de l'intelligence est intimement lié à la construction de comportements intentionnels, les intentions étant liées à la culture (Bruner, 2000). Dans le contexte des approches interactionnistes de la construction de connaissances, ce point de vue nous amène à nous intéresser, au rôle qui joue la prise en compte par l'enseignant, des différences culturelles et linguistiques entre enfants dans les activités des sciences expérimentales :

- Certaines caractéristiques du fonctionnement social de l'enfant peuvent-elles favoriser ou gêner les constructions des savoirs scientifiques au cours d'une activité scolaire?

Au côté des interactions maître-élève, les interactions entre pairs paraissent exercer une influence notable dans ces processus de changement. Sous certaines conditions (la nature et la difficulté des tâches notamment), les rencontres égalitaires ou légèrement dissymétriques infléchissent plus favorablement les fonctionnements individuels que les périodes d'activité autonome. Ce type d'échanges constituent un jeu 
social complexe, la relation entre les participants supposant la circulation d'affects (positifs ou non), tant à l'égard du partenaire que de la situation d'observation, la poursuite de buts sociaux (dominer l'autre et exercer un leadership, favoriser le partage des responsabilités pour résoudre les problèmes, etc.) et la négociation de rôles qui tissent des climats interactionnels singuliers (Gilly, Roux, \& Trognon, 1999 ; Kerbrat-Orecchioni, 1998).

La répartition des rôles, avec permutation des enfants dans les différents rôles, va contraindre les enfants à verbaliser ce qu'ils font. Les compétences et les performances d'un élève dépendent à la fois des compétences et des performances des autres élèves de sa classe et de ses possibilités propres de résister à cette influence. Les deux raisons qui justifient le fait que les interactions soient d'une grande importance pour la didactique des langues sont le fait qu'elles régulent l'enseignement apprentissage (selon des règles qu'il convient de décrire et d'analyser), mais aussi le fait qu'elles sont elles-mêmes objets d'apprentissage.

Dans quelle mesure les interactions entre pairs contribuent-elles au développement de la compétence langagière de l'enfant allophone?

\section{Population de l'étude et description de l'activité}

La recherche étant en cours de réalisation et ayant un caractère exploratoire, cette communication présentera les premiers résultats issus des observations menées dans deux classes appartenant à des groupes socioculturels linguistiques distincts : une classe d'élèves se trouvant à Cayenne (de langue maternelle créole haïtien) et l'autre dans un site isolé sur le fleuve Oyapock, à Camopi (élèves parlant l'émérillon, langue amérindienne).

La description des interactions étant présentée à travers une approche comparative, nous avons conservé un nombre important de constantes : le même intervenant, le chercheur (pour éviter les interprétations liées au style d'enseignement du professeur); la même activité - à caractère scientifique (les sciences présentant une valeur supra-communautaire); des classes mixtes, de 24 élèves âgés de 6 ans (+/- 6 mois) ; le même moment de la journée, de $09 \mathrm{~h} 30$ à $11 \mathrm{~h} 00$ (afin de restreindre les écarts de performances qui pourraient être dus aux rythmes chrono-biologiques); le déroulement de l'activité a eu lieu dans la salle de classe, avec le même matériel didactique.

Suite à l'entretien avec les enseignants des deux classes, une deuxième variable a été considérée, la présence (ou non) de prérequis en lien avec l'activité scientifique proposée.

Le domaine des sciences a été choisi en partant du présupposé selon lequel les sciences, par leurs prétentions à l'universalité, constitueraient un terrain privilégié pour réunir des communautés pouvant fonctionner sur des valeurs très différentes. Les explications scientifiques étant par essence transculturelles, un travail sur celles-ci nous semble pouvoir permettre de dépasser les particularismes culturels.

La séquence d'activité (déclinée en trois séances réalisées à deux semaines d'écart chacune) portait sur la mise en évidence des propriétés de l'air (invisible, incolore, inodore, résistant) et la construction d'un objet (une fusée à réaction) à partir de fiches de fabrication. Nous avons fait l'hypothèse qu'une activité scientifique portant sur la réalisation d'un objet technique faciliterait la communication entre élèves et entre élèves/intervenant et pourrait constituer un déclencheur de collaboration.

\section{Les exigences du corpus}

S'agissant des productions mobilisant les savoirs et la langue orale (l'unité d'analyse étant la connaissance partagée, négociée, appropriée, située dans les situations d'apprentissage mises en place par le tuteur), il convient non seulement de filmer les séquences identifiées, mais aussi de transcrire les données selon une approche ethnométhodologiste avec des règles qui ne dénaturent pas les situations interactionnelles et les productions réelles, tout en prenant en compte la manière dont le matériel verbal 
est produit : répétions, régulateurs, structures d'hésitation, etc. Au fur et à mesure de la progression de l'analyse, des retours aux données du corpus ont été faites pour affiner la transcription (cf. tableau 1).

Dans le tableau des transcriptions ci-contre, nous avons surligné en gris les interventions du professeur pour mettre ainsi en évidence les échanges qui seront initiés dans les deux groupes de classe ; cette analyse nous semble pertinente dans la mesure où l'initiateur de l'échange, le professeur, est le même et le scenario détaillé de l'activité est également le même. Il est à noter que certaines interventions du professeur seront spécifiques à chaque groupe en fonction des interventions réactives des élèves (demande de précisions, relances, etc.) ; toutefois le tuteur va revenir ensuite au déroulement prévu par l'activité, en respectant les interventions initiatives prévues dans le scenario.

L'analyse des transcriptions a été faite en utilisant une démarche analytique basée sur les dynamiques interactives, les logiques dialogiques, épistémiques, et les reformulations (Kerbrat-Orecchioni, 1998).

Par l'analyse envisagée, il s'agit de rendre compte de la dynamique interactive qui se crée au sein de la classe (élèves-élèves, professeur-élèves) au cours de l'interaction dans ses diverses composantes langagières et pratiques et des concepts et savoirs acquis par les élèves.

\begin{tabular}{|c|c|c|c|c|c|}
\hline & $\mathbf{N}^{\circ}$ & Echanges Cayenne & Echanges Camopi & $\mathbf{N}^{\circ}$ & \\
\hline \multirow[t]{3}{*}{ 2' } & 1.11 & \multicolumn{2}{|c|}{$\begin{array}{l}\text { T: Les enfants, quand on souffle, qu'est ce qu'il sort de notre } \\
\text { bouche? }\end{array}$} & 1.08 & $1{ }^{\prime} 25$ \\
\hline & 1.12 & $\begin{array}{l}\text { Les enfants commencent à } \\
\text { souffler. }\end{array}$ & A1 : De l'air. & 1.09 & \\
\hline & 1.13 & $T:$ Allez, soufflez tous! & A2 : Du vent. & 1.10 & \\
\hline \multirow[t]{3}{*}{$22^{\prime 20}$} & 1.14 & A $10:$ Du vent. & A3 : De l'air & 1.11 & \\
\hline & 1.15 & A 19: Du souffle. & & & \\
\hline & 1.16 & $\begin{array}{l}\text { T: Oui, du vent, du souffle, de } \\
\text { l'air. }\end{array}$ & & & $11^{\prime} 45$ \\
\hline \multirow[t]{4}{*}{$2 ’ 25$} & 1.17 & \multicolumn{2}{|c|}{$T:$ Et d'après vous, d'où sort cet air? } & 1.12 & \\
\hline & 1.18 & $\begin{array}{l}\text { De là-dedans (en montrant le } \\
\text { ventre). }\end{array}$ & Tout le monde souffle! & 1.13 & \\
\hline & 1.19 & De l'estomac. & Tous les enfants soufflent. & 1.14 & \\
\hline & 1.20 & Du ventre. & D'où vient le souffle? l'air? & 1.15 & \\
\hline \multirow[t]{5}{*}{ 2’40 } & 1.21 & De là dedans, du ventre... & De la gorge. & 1.16 & \\
\hline & & & De le nez. & 1.17 & \\
\hline & & & De la bouche. & 1.18 & \\
\hline & & & $\begin{array}{l}\text { L'enseignant souffle sur la } \\
\text { pomme de la main. }\end{array}$ & 1.19 & 2’10 \\
\hline & & & $\begin{array}{l}\text { Ils imitent l'enseignant et } \\
\text { soufflent sur la pomme de la } \\
\text { main }\end{array}$ & 1.20 & \\
\hline
\end{tabular}

Tableau 1 : Extrait des transcriptions au cours de la première séance -à une même intervention de l'adulte, les réponses des élèves des deux classes considérées.

Les échanges adulte-enfant ont été intégralement transcrits en y ajoutant des aspects non verbaux (gestes, manipulations, mimiques). En effet, les composantes non verbales dans la communication adulte-enfant dans des contextes de jeu sont très importantes (par exemple, les gestes d'encouragement et les mimiques de satisfaction chez l'adulte, la réalisation d'actions chez l'enfant).

\section{Le modèle d'analyse et les premiers résultats}

Nous avons procédé au découpage du corpus en séquences en se basant sur les recherches réalisées par Ailincai (2005) et François et Weil-Barais (2003), qui soulignent l'importance de la prise en compte des conduites des interactants et du contenu informationnel dans la construction des connaissances en cours 
d'interaction (reconnaître et démontrer la présence de l'air / définir ou mettre en évidence quelques propriétés : invisible, incolore, inodore, il permet la respiration / observer l'action de l'air sur les objets / mener et raconter des expériences simples / fabriquer un objet technologique simple).

Deux types d'analyses, que nous présentons succinctement, ont été conduites; elles concernent (1) la nature des interactions et (2) la structure des échanges professeur-enfant(s) et enfants-enfants.

Pour répondre à notre questionnement et rendre compte de la nature des interactions, nous nous sommes proposés dans un premier temps d'analyser les types de relations qui se sont instaurées au cours de la situation d'apprentissage entre les différentes composantes de l'interaction que sont les élèves, le tuteur, le savoir et l'instrument, dans ce contexte particulier, plurilingue et pluriculturel, en se basant sur le modèle proposé par Bernard (2006) (cf. figure 1).

L'analyse de la structure des échanges s'inspire des travaux relatifs à l'analyse conversationnelle. Les échanges ont été identifiés et caractérisés selon les critères définis par Kerbrat-Orecchioni (1998) : échanges restreints, échanges étendus, échanges tronqués. Cette analyse nous intéresse pour l'analyse de l'évolution de la structure des échanges entre le professeur et les élèves au cours de trois activités menées avec chaque classe. La proportion d'échanges étendus (caractérisés par plus de trois tours de parole) est un bon indicateur de l'attention conjointe des interlocuteurs et c'est à ce titre que nous l'avons utilisée. De même les échanges tronqués qui se caractérisent par une absence de réaction à une intervention du partenaire, indiquent des difficultés dans la relation éducative.

\subsection{La nature de interactions}

Le codage de l'activité transcrite a été réalisé en s'inspirant du modèle du carré médiatique de Bernard (2006) et du modèle SKIT de Bernard \& Baker (2007) (cf. figure 1). Dans ces modèles (Adulte/Enfant chez Bernard ou Élèves/Professeur chez Bernard \& Baker) la situation interactive évolue, elle n'est plus dyadique mais d'emblée polyadique avec de nombreuses modulations possibles : c'est l'instrument (l'artefact à réaliser), la situation (collaborative ou pas) et l'espace ouvert par les interactions multiples qui donnent à l'enfant de nouvelles possibilités pour apprendre. La séquence d'interaction la plus complète est obtenue lorsque la médiation humaine et instrumentale, entre l'élève et le savoir, fonctionne pleinement.

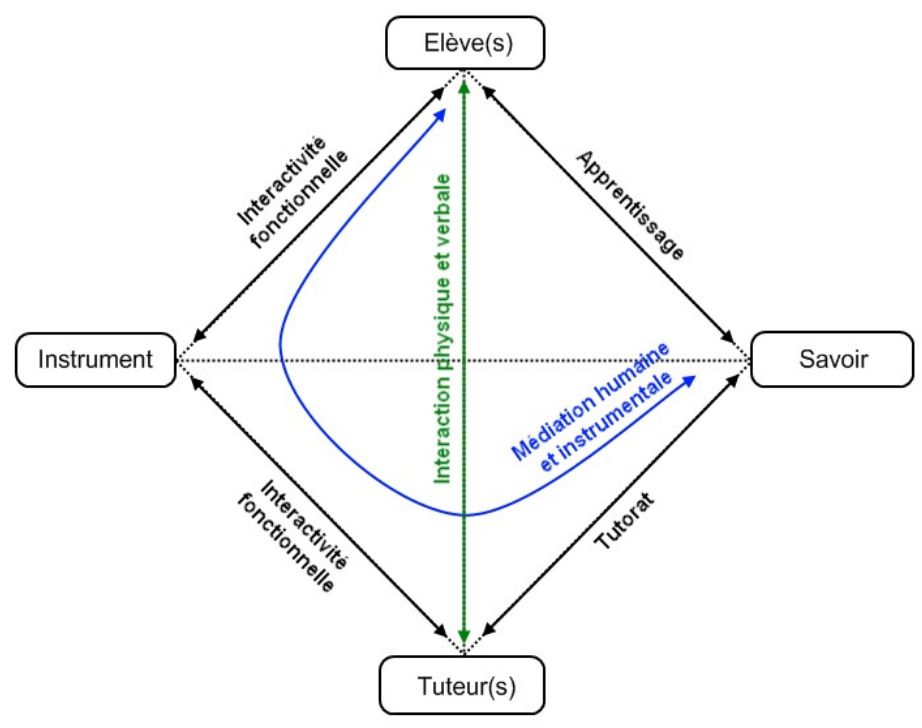

Figure 1 : Le Carré médiatique (Bernard, 2006) 
Dans ce cas, les quatre pôles du carré sont «opérationnels ». Le tuteur, au moyen de l'instrument que l'élève manipule, va faire ressortir le contenu de savoir médiatisé par cet instrument. Le carré médiatique, tel qu'il est schématisé ci-dessus, rend compte d'un certain état de la situation médiatique, cet état pouvant prendre différentes formes au cours de l'activité. Les caractéristiques de la situation médiatique vont évoluer en fonction des conduites des deux groupes de partenaires qu'elle solidarise, le(s) élève(s) et le(s) tuteur(s).

En s'appuyant sur la description proposée par les auteurs cités (et en conservant ses éléments constitutifs : savoir, instrument, enseignant, élèves), nous proposons une grille de codage et d'analyse (cf. Tableau 2) qui distingue par ailleurs :

l'initiateur de l'interaction (tuteur ou apprenants),

les conduites marquantes des élèves, qui peuvent avoir un impact sur la structure interactionnelle (l'attention conjointe, la réponse collective, l'absence d'attention, les problèmes de discipline)

- ainsi que la nature du savoir dont il est question dans l'échange -qui peut être le savoir en jeu ou bien un autre savoir sans rapport avec l'objectif de l'activité.

\begin{tabular}{|c|c|c|}
\hline \multicolumn{3}{|c|}{ Interaction avec le tuteur } \\
\hline \multirow[t]{2}{*}{ TASI } & \multirow[t]{2}{*}{$\begin{array}{l}\text { Tuteur-Apprenant- } \\
\text { Savoir en jeu- } \\
\text { Instrument }\end{array}$} & $\begin{array}{l}\text {-v (verbal) Ex. T : Comment pourrais-tu mettre l'air en mouvement ? } \\
\text { A : Je fais comme ça... L'apprenant agite l'éventail pour mettre l'air en } \\
\text { mouvement, et verbalise l'action }\end{array}$ \\
\hline & & $\begin{array}{l}\text {-n (non verbal) Situation identique, l'apprenant réalise la tâche, mais } \\
\text { sans verbalisation. }\end{array}$ \\
\hline \multirow[t]{2}{*}{ TAS } & \multirow{2}{*}{$\begin{array}{l}\text { Tuteur-Apprenant- } \\
\text { Savoir en jeu }\end{array}$} & -v (verbal) Ex. $\mathbf{T}$ : d'où vient cet air ? / $\mathbf{A}$ : de mon ventre \\
\hline & & -n (non verbal) Situation identique, $\mathbf{A}:$ il montre \\
\hline \multirow[t]{2}{*}{ TAas } & \multirow{2}{*}{$\begin{array}{l}\text { Tuteur-Apprenant- } \\
\text { autre savoir }\end{array}$} & $\begin{array}{l}\text {-v (verbal) Ex. } \mathbf{T} \text { : est-ce qu'on peut vivre sans respirer ? / } \mathbf{A}: \text { Oui, on } \\
\text { met un casque... }\end{array}$ \\
\hline & & $\begin{array}{l}\text {-n (non verbal) Situation identique, l'enfant affirme avec un hochement } \\
\text { de tête. }\end{array}$ \\
\hline \multirow[t]{2}{*}{ TAI } & \multirow{2}{*}{$\begin{array}{l}\text { Tuteur-Apprenant- } \\
\text { Instrument }\end{array}$} & -v (verbal) Ex. T: A quoi sert une flute? $\mathbf{A}:$ A faire de la musique. \\
\hline & & $\begin{array}{l}\text {-n (non verbal) Situation identique, l'enfant joue avec l'instrument ou } \\
\text { lui donne une autre utilisation. }\end{array}$ \\
\hline \multicolumn{3}{|c|}{$\begin{array}{l}\text { Conduites des interactants qui caractérisent certains temps de l'interaction ou qui sont susceptibles } \\
\text { de la modifier }\end{array}$} \\
\hline & Attention Conjointe & Les apprenants observent un phénomène pour avoir une réponse. \\
\hline & Réponse Collective & Plusieurs apprenants répondent aux sollicitations de l'adulte. \\
\hline & $\begin{array}{l}\text { Réponse Collective - } \\
\text { problèmes de discipline }\end{array}$ & $\begin{array}{l}\text { Les apprenants répondent bruyamment en dérangeant l'activité et/ou } \\
\text { interrompent l'interaction. }\end{array}$ \\
\hline & $\begin{array}{l}\text { Absence d'attention - } \\
\text { problèmes de discipline }\end{array}$ & Le tuteur interrompt pour gérer les situations de discipline \\
\hline \multicolumn{3}{|c|}{ Interaction entre les élèves } \\
\hline \multirow[t]{2}{*}{ AAI } & \multirow{2}{*}{$\begin{array}{l}\text { Apprenant-Apprenant- } \\
\text { Instrument }\end{array}$} & -v (verbal) Ils discutent au sujet du matériel ; pas de savoir \\
\hline & & $\begin{array}{l}\text {-n (non verbal) Ils manipulent le matériel sans se préoccuper de la } \\
\text { tâche demandée ; pas de savoir }\end{array}$ \\
\hline AAas & $\begin{array}{l}\text { Apprenant-Apprenant- } \\
\text { autre savoir }\end{array}$ & -v (verbal) Ils échangent entre eux au sujet d'un autre savoir. \\
\hline AAS & $\begin{array}{l}\text { Apprenant-Apprenant- } \\
\text { Savoir en jeu }\end{array}$ & -v (verbal) Ils échangent entre eux au sujet du savoir en jeu. \\
\hline \multirow[t]{2}{*}{ AASI } & \multirow{2}{*}{$\begin{array}{l}\text { Apprenant-Apprenant- } \\
\text { Savoir en jeu - } \\
\text { Instrument }\end{array}$} & -n (non verbal) Ils réalisent ensemble une action liée au savoir en jeu. \\
\hline & & $\begin{array}{l}\text {-v (verbal) Ils réalisent ensemble une action et échangent entre eux au } \\
\text { sujet du savoir en jeu. }\end{array}$ \\
\hline
\end{tabular}

Tableau 2 : Modèle de codage des interactions, d'après Bernard (2006)

Ces critères nous permettront entre autres de rendre compte de la dynamique interactive dans les groupes considérés; ils apparaissent dans la figure 2, où trois espaces sont identifiés: l'espace interactif 
comprenant les échanges initiés par le tuteur, les conduites des apprenants ayant une influence sur le déroulement de l'interaction (l'attention conjointe, la réponse collective, l'absence d'attention, les problèmes de discipline) et l'espace interactif comprenant les échanges initiés par les apprenants.

Ce type de codage, représenté sous forme de diagramme nous a permis l'élaboration des tracés pour les deux classes et pour tous les échanges durant l'activité (dans cet article nous présentons seulement l'analyse la première séance). Une lecture rapide du diagramme met en évidence des spécificités facilement repérables, pour les deux classes; nous reviendrons sur ces aspects lors de l'analyse des résultats.

Afin de pouvoir conserver les mêmes codes pour la suite de notre recherche (lors de l'analyse des interactions au sein de la famille), nous avons fait le choix d'identifier les interactants comme suit: le professeur est désigné par « $\mathrm{T}$ » (tuteur) et les élèves sont désignés par « $\mathrm{A} »($ apprenant).

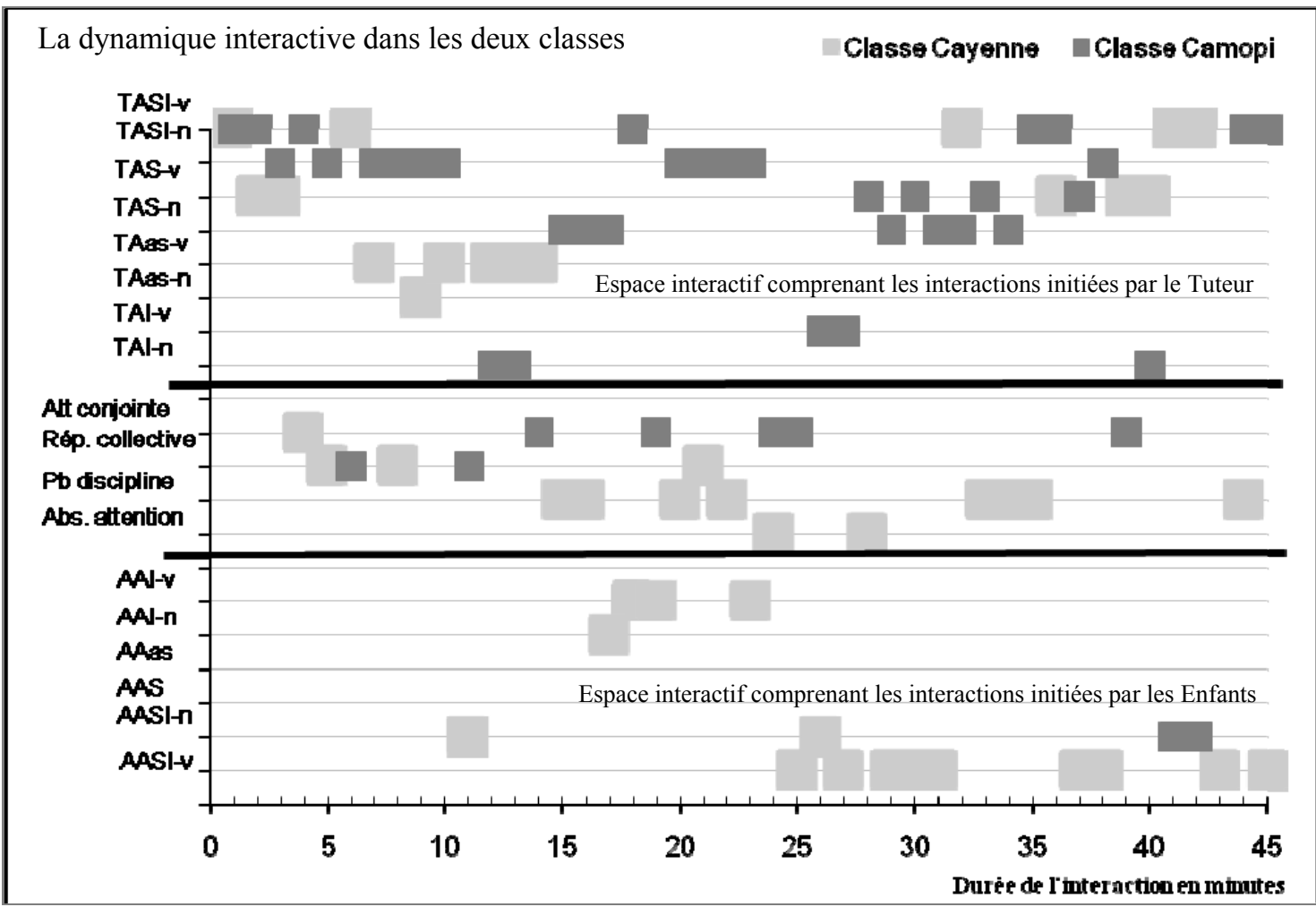

Figure 2 : Diagramme des deux classes lors de la première activité.

\subsection{La structure des échanges professeur-enfant(s) et enfants-enfants}

En s'inspirant des travaux de Kerbrat-Orecchioni (1998), nous avons procédé dans un deuxième temps à une transcription schématisée de l'analyse du corpus, la carte dialogique de l'interaction (Ailincai, 2007), qui fait ressortir les constituants de chaque interaction : les séquences, le type des échanges (restreint, tronqué, étendu), les interventions, la durée de l'interaction, l'initiateur de l'échange, etc. (cf. figure 3).

Ce mode d'analyse nous a permis d'identifier facilement s'il y avait changement dans la structure de l'interaction (type d'échange, initiateur de l'échange, prépondérance du verbal et du non verbal, etc.) sur les trois séances mises en place dans chaque classe. Ces résultats ont besoin d'être complétés et confirmés par des analyses plus fines (par exemple, du type analyse interlocutoire). Lors de la première séance cet 
extrait de la carte de l'interaction met en évidence deux échanges tronqués et un échange restreint, alors que lors des séances suivantes nous constatons la présence des échanges restreints et étendus et même un travail en autonomie lors de la troisième séance (cf. figure 3).

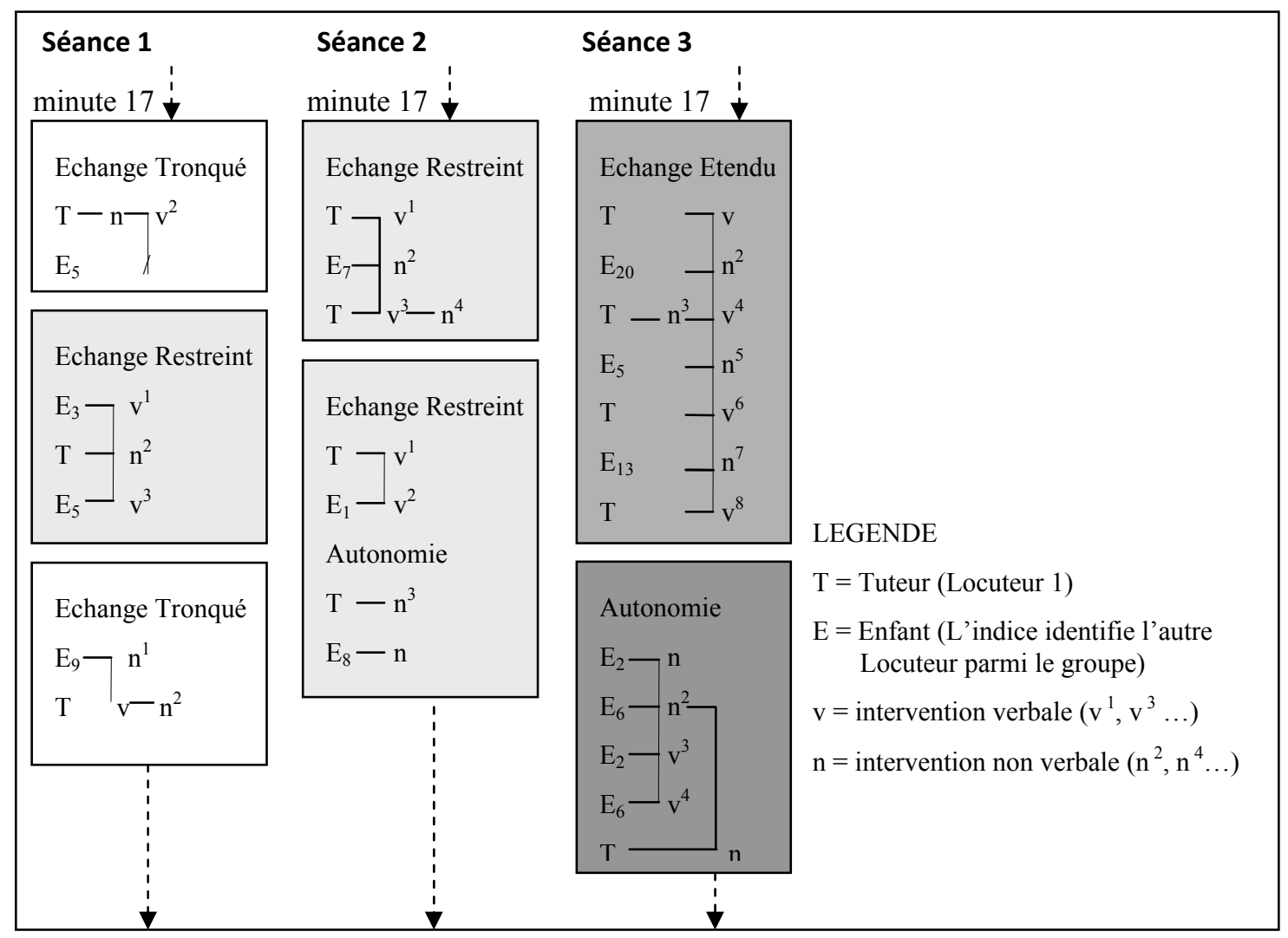

Figure 3 : Extrait de la carte dialogique des interactions, classe Cayenne, les trois séances, minute 17

En analysant l'ensemble des échanges sur les trois séances menées dans les deux classes (cf. Tableau 3), nous constatons que les deux groupes d'élèves conservent une spécificité sur le plan de la structure de l'interaction (lecture verticale du tableau pour chaque classe et chaque séance), spécificité qui les différencient et, par ailleurs, une évolution de la qualité des échanges se fait remarquer sur les trois séances et pour chacune des deux classes (lecture horizontale du tableau), évolution qui les réunit.

Concernant la structure de l'interaction nous remarquons des différences entre les deux classes au cours des trois séances :

l'une est caractérisée par une forte présence d'échanges tronqués sur l'ensemble des échanges de la séance (18 échanges tronqués lors de la première séance contre deux échanges étendus et 14 et 12 échanges tronqués lors des séances suivantes, contre respectivement cinq et cinq échanges étendus),

alors que l'autre classe présente un important nombre d'échanges restreints $(21,20$ et 18 échanges restreints sur les trois séances, contre respectivement un, cinq et cinq échanges étendus).

En regardant le corpus, cela peut se traduire par la présence des problèmes d'attention conjointe et de discipline dans la classe caractérisée par une forte présence d'échanges tronqués (beaucoup d'interventions initiatives de la part des élèves et sans rapport avec le savoir en question) et, pour l'autre classe, de langue maternelle émerillon, par un manque d'interventions initiatives de la part des élèves, ces derniers se contentant de répondre d'une manière brève aux questions du professeur (interventions réactives et en rapport avec le savoir traité). 


\begin{tabular}{|c|l|c|c|c|}
\cline { 2 - 4 } \multicolumn{2}{c|}{} & Séance 1 & Séance 2 & Séance 3 \\
\hline \multirow{3}{*}{$\begin{array}{c}\text { Elèves de langue } \\
\text { maternelle créole haïtien }\end{array}$} & Echange Etendu & 02 & 05 & 05 \\
\cline { 2 - 4 } & Echange Tronqué & 18 & 14 & 12 \\
\cline { 2 - 4 } & Echange Restreint & 11 & 12 & 11 \\
\cline { 2 - 4 } & Séance en autonomie & 03 & 02 & 14 \\
\hline \multirow{3}{*}{$\begin{array}{c}\text { Elèves de langue } \\
\text { maternelle émerillon }\end{array}$} & Echange Etendu & 01 & 05 & 05 \\
\cline { 2 - 5 } & Echange Tronqué & 03 & 20 & 18 \\
\cline { 2 - 5 } & Echange Restreint & 21 & 05 & 20 \\
\cline { 2 - 5 } & Séance en autonomie & 04 & 03 & 02 \\
\hline
\end{tabular}

Tableau 3: Nombre des échanges les plus marquants (étendus, restreints, tronqués et séances en autonomie), au cours des trois activités dans les deux classes (durée de chaque activité : 45 min.)

Concernant la qualité des échanges de l'interaction, nous constatons une évolution au fil des trois séances pour les deux classes : les échanges tronqués diminuent pour les deux classes, en faveur des échanges étendus et des temps en autonomie (ces derniers augmentent visiblement), ce qui peut traduire une amélioration de la communication professeur-enfant et enfants-enfants.

\section{Discussion et conclusion}

Le diagramme, commun aux deux classes, permet de caractériser chaque interaction en fonction des séquences qui la constituent - relativement au codage qui en a été fait -, mais aussi en fonction de l'ordre d'enchaînement et de la durée de ces séquences tout en permettant une analyse comparative de deux groupes considérés.

Une première lecture du diagramme fait apparaitre des disparités selon l'initiateur du groupe. On peut déjà noter que pour la classe de Camopi (identifiée dans le diagramme par la couleur foncée) le tuteur a été l'initiateur de l'ensemble des échanges, avec une exception vers la fin de l'activité qui d'ailleurs se caractérisait par une séquence d'autonomie laissée aux enfants. Concernant la classe de Cayenne, si dans le premier quart d'heure de l'activité les échanges sont initiés par le tuteur, nous remarquerons que pour le reste de l'activité les élèves ont été très impliqués, en initiant la grande majorité des échanges. Toutefois, cette implication est souvent alternée avec des problèmes de discipline. Sur ce point l'autre classe, celle de Camopi, apparaît souvent en situation d'attention conjointe avec très peu de réponses collectives.

Par ailleurs dans le groupe de Camopi, contrairement au groupe de Cayenne, les échanges ont toujours portés sur les savoirs en jeu ; cela peut s'expliquer par le fait que les échanges ont été initiés par le tuteur, selon un scénario précis. Ce dernier a connu certains ajustements dans l'autre groupe où, en dehors des situations imprévisibles générées par les interventions initiées par les enfants, plusieurs échanges ont porté sur d'autres savoirs, liées à la visite que les enfants de Cayenne avaient faite à la «Cité de l'Espace » à Kourou.

Il est à noter que les évaluations réalisées après coup montrent que l'objectif de la séance a été atteint pour les deux groupes d'élèves. Cette analyse étant la première d'une série d'observations (plusieurs activités ont été filmées), nous nous garderons bien d'une conclusion généralisante. Les caractéristiques observées pourraient s'expliquer par les spécificités socioculturelles des classes étudiées, mais aussi par le style d'enseignement de l'enseignant titulaire de la classe.

En conclusion, nous serions tentés de dire que, dans le cas précis de la situation observée (qui présente la spécificité d'avoir un nombre important de constantes -les principales étant la présence du même tuteur et d'un scénario d'activité bien étudié et maittrisé-, et avec seulement deux variables -les apprenants et les prérequis dans une des classes-), nous remarquons des spécificités quant au format interactif de l'activité, par rapport aux situations interactives classiques (Kerbrat-Orecchioni, 1998). Ces spécificités se manifestent tant sur le plan de l'initiateur de l'échange, que sur la participation à l'activité en termes d'attention conjointe. 
Par ailleurs la présente communication n'a pas d'autre prétention que de proposer une grille de codage et un modèle de transcription qui synthétise un nombre important de données permettant de caractériser une interaction. Le codage proposé nous apporte des informations concernant la présence dans l'échange du savoir (ou du savoir faire) mis en jeu, ainsi que de la dynamique interactive qui caractérise une activité d'enseignement/apprentissage.

La transcription, que nous avons appelé carte dialogique de l'interaction, se montre efficace surtout pour les analyses comparatives des interactions. Elle peut nous renseigner sur l'évolution des échanges langagiers en termes de longueur (pour les échanges étendus), de qualité de la tutelle (baisse importante des échanges tronqués), d'initiateur de l'échange (dans notre contexte le plus souvent le parent), de durée (des échanges, des séquences et des interactions), etc.

Nous rappelons que nous nous situons dans une démarche d'investigation (recherche d'une cohérence et des points communs dans la dynamique d'apprentissage entre les différents groupes d'élèves - locuteurs de langues amérindiennes et du créole guyanais-) mais également dans une démarche comparative, afin de mettre en évidence des éventuelles particularités analysées dans leur contexte (valeur heuristique).

La recherche étant exploratoire, nous faisons l'hypothèse d'une variabilité de la dynamique interactionnelle entres les six classes, tant du côté des élèves que du coté du tuteur "unique" (qui, au vu des premiers résultats, adapte sa conduite en fonction des besoins des élèves). L'éventuel écart entre les structures interactionnelles des classes considérées pourrait s'expliquer entre autres, par les spécificités des styles éducatifs pratiqués au sein des groupes socioculturels auquel appartiennent les élèves (Pourtois, 1979 ; Ogbu, 1981), mais également par les différences entres les styles interactifs pratiqués par les enseignants titulaires.

\section{Références bibliographiques}

Ailincai, R. (2005). Un dispositif d'éducation parentale. Sensibilisation des parents à leur rôle d'accompagnateur de leur enfant dans le cadre d'un musée à caractère scientifique et technique. Thèse de doctorat Université Paris 5.

Ailincai, R. (2007). «La carte dialogique de l'interaction», un exemple d'analyse séquentielle. Actualités psychologiques. Lausanne, Suisse : Institut de psychologie de l'Université de Lausanne

Alby, S. et Léglise, I. (2006). L'enseignement en Guyane et les langues régionales. Réflexions sociolinguistiques et didactiques. Marges Linguistiques, 10, 245-261.

Alby, S. et Léglise, I. (2007b). La place des langues des élèves en contexte guyanais. Quatre décennies de discours scientifiques. In S. Mam Lam Fouck (ed), Comprendre la Guyane d'aujourd'hui, Cayenne : Ibis Rouge Editions, 439-452.

Bachelard, G. (1938). La formation de l'esprit scientifique. Paris : J. Vrin.

Bernard, F-X. (2006). L'impact cognitif des dispositifs médiatiques sur les enfants d'âge préscolaire en situation d'apprentissage avec un adulte: Étude d'un cas de simulateur informatique dans le contexte d'une exposition scientifique. Thèse de doctorat Université Paris 5.

Bernard, F.-X. \& Baker, M. (2007). Deliverable D4.1: Research evaluation plan, LEAD project, Specific Targeted Research Project, IST-2005-028027. http://www.lead2learning.org/assets/projectsite/Deliverables/LEAD 028027 _D4.1_ResearchEvaluationPlan_v1_20070530nk.pdf

Bruner, J. (1983). Le développement de l'enfant. Savoir faire, savoir dire. Paris : Presses Universitaires de France.

François, A. \& Weil-Barais, A. (2003). Élaboration des connaissances relatives à un dispositif technique dans un contexte d'interactions parent-enfant. Bulletin de Psychologie, 56 (4), 509-519.

Gilly, M., Roux, J.P., \& Trognon, A. (Eds.) (1999). Apprendre dans l'interaction. Nancy : Presses Universitaires de Nancy et Publications de l'université de Provence.

Kerbrat-Orecchioni, C. (1998). Les interactions verbales, tome 1. Paris : Armand Colin.

Leconte, F. et Caïtucoli, C. (2003). Contact de langues en Guyane : une enquête à Saint-Georges de l'Oyapock. In J. Billiez (ed), Contacts de langues : modèles, typologies, interventions, Paris : L'Harmattan, 37-59. 
Léglise, I. (2004). Langues frontalières et langues d'immigration en Guyane française. Glottopol, 4, 108-124.

Léglise, I. (2005). Contacts de créoles à Mana (Guyane française) : répertoires, pratiques, attitudes et gestion du plurilinguisme. Etudes Créoles, 28, 23 - 57.

Léglise I. \& Puren L., (2005), «Usages et représentations linguistiques en milieu scolaire guyanais », Ecole et éducation, Univers créoles, 5, 67-90.

Léglise, I. (2007). Des langues, des domaines, des régions. Pratiques, variations, attitudes linguistiques en Guyane. In I. Léglise et B. Migge (eds), Pratiques et représentations linguistiques en Guyane : regards croisés. Paris : IRD Editions.

Léglise, I., Migge, B. (2007). Pratiques et représentations linguistiques en Guyane : regards croisés. Paris : IRD Editions.

Li, J. (2002). Learning models in different cultures, New Directions for Child and Adolescent Development, 96, 4563.

Ogbu, J. (1981). School Ethnography : A multilevel approach, in Anthropology and Education Quarterly, 12, 38-60.

Pourtois, J-P., \& Desmet, H. (2004). L'éducation implicite. Paris : Presses Universitaires de France

Vygotski, L. S. (1934). Pensée et langage, Traduction de F. Seve, Paris : Messidor / Editions sociales

Weil-Barais, A. (2004). Les apprentissages scolaires. Rosny sous bois : Bréal Éditeur.

Weil-Barais, A., Bernard, F.-X., Cho, H.Y., \& Lacroix, F. (2009). Développement des compétences cognitives des enfants dans des contextes d'interaction éducative. TRANEL (Travaux neuchâtelois de linguistique), 49, 169-186.

\footnotetext{
${ }^{1}$ Aux Etas Unis, il a été montré que les enfants d'origine chinoise (qui ont des meilleures performances scolaires), manifestent des conceptions très différentes de celles des enfants issus de l'immigration européenne (Li, 2002). Pour les premiers l'apprentissage est lié à l'effort, à la volonté, à la réflexion, au dépassement de soi, au travail personnel ; pour les seconds il est lié aux compétences et habiletés, aux exercices, à la motivation, aux moyens disponibles. Par ailleurs, pour les asiatiques, c'est le sérieux et l'investissement personnel qui prédominent, alors que chez les autres, c'est le caractère ludique et social: on apprend en s'amusant, on va à l'école pour rencontrer des copains. La perception des finalités des apprentissages diffèrent également: le développement personnel pour les uns, la connaissance du monde et des impératifs sociaux pour les autres.
} 